\title{
Patterns of Antimicrobial and Multi Drug Resistance in E. coli and Salmonella Isolates of Commercial and Non-Commercial Poultry
}

\author{
Asghar Ali Kamboh ${ }^{1 *+}$, Tarique Ali Rind ${ }^{1+}$, Muhammad Ammar Khan ${ }^{2}$, Rehana Burriro ${ }^{3}$, Riaz Ahmed Leghari ${ }^{4}$ and \\ Kanwar Kumar Malhi ${ }^{1}$ \\ ${ }^{1}$ Department of Veterinary Microbiology, Faculty of Animal Husbandry and Veterinary Sciences, Sindh Agriculture \\ University, 70060 Tandojam, Pakistan \\ ${ }^{2}$ Department of Food Science and Technology, University College of Agriculture and Environmental Sciences, The Islamia \\ University of Bahawalpur, 63100 Bahawalpur, Pakistan \\ ${ }^{3}$ Department of Veterinary Pharmacology, Faculty of Animal Husbandry and Veterinary Sciences, Sindh Agriculture \\ University, 70060 Tandojam, Pakistan \\ ${ }^{4}$ Department of Veterinary Medicine, Faculty of Animal Husbandry and Veterinary Sciences, Sindh Agriculture University, \\ 70060 Tandojam, Pakistan \\ *For correspondence: drasgharkamboh@yahoo.com \\ ${ }^{\dagger}$ Contributed equally to this work and are co-first authors \\ Received 30 January 2021; Accepted 06 April 2021; Published 10 June 2021
}

\begin{abstract}
The extensive and abuse of antibiotics have contributed to the universal spread of antibiotic resistance (AR). Commercial poultry birds harbor more antibiotic-resistant microorganisms than the backyard chicken, but the status is not published in quails. This study was designed to investigate the status of AR microbiota in C0: backyard chickens, C1: commercial broiler, Q0: backyard/wild quails and Q1: commercial quails $(\mathrm{n}=20)$. Escherichia coli (E. coli) and Salmonella isolates from carcass and ceca of these chickens and quails were investigated for incidence and extent of AR using disk diffusion method. The results of overall microbiota of the experimental birds revealed that $\mathrm{C} 1$ showed a greater $(P<0.01) \mathrm{AR}$ as compared with $\mathrm{C} 0$ for ampicillin, chloramphenicol, ciprofloxacin, gentamicin, neomycin, norfloxacin, oxytetracycline, and sulfamethoxazole, with about 57.39, 57.24, 38.78, 62.92, 36.51, 67.61, 55.83 and 55.68\% greater incidence of AR, respectively. Similarly, Q1 also exhibited a greater $(P<0.01)$ AR than Q0 for these antibiotics, with about 65.59, 58.44, 54.38, 54.38, 55.68, 51.62, 54.87 , and $64.93 \%$, respectively. Moreover, the results of individual microbial numbers of both the pathogenic bacterial isolates from $\mathrm{C} 1$ and Q1 exhibited a higher $(P<0.01)$ AR for all tested antimicrobials than those isolated from C0 and Q0. Additionally, the E. coli and Salmonella isolates of $\mathrm{C} 1$ and Q1 were more $(P<0.05)$ multi drug resistant (MDR) as compared with $\mathrm{C} 0$ and Q0. Furthermore, the extent of AR was greater in E. coli and Salmonella isolates of C1 and Q1 in contrast to those of C0 and Q0. Finally, C1 and Q1 harbored a greater number of MDR bacterial species than those in C0 and Q0 thus may act as risk factors for antimicrobial dissemination. (C) 2021 Friends Science Publishers
\end{abstract}

Keywords: Antimicrobial resistance; Multidrug resistance; Backyard poultry; Quail; Pathogenic bacteria

\section{Introduction}

The non-judicious use of antimicrobial agents in poultry feed has been associated with an increased incidence of antibiotic resistance (AR) in the microorganisms of poultry origin. Commercial poultry farming is highly profitable because it produces poultry meat with the least investment within 5-6 weeks. Poultry farmers observe intense care in commercial broiler farming, and particularly in the developing countries, such as Pakistan, in-feed antibiotic drugs are prevalent for preventing a variety of poultry diseases (Kamboh et al. 2018a).
Although antibiotics are essential for disease prevention and control, these have also found another use as growth promoters in the poultry industry (Obajuluwa et al. 2021; Schwarz et al. 2001). A number of recently published reports indicated that the meat gained from the broilers reared on conventional antibiotic mixed feeds harbored high counts of AR bacteria as compared to the one obtained from organically reared chickens (Miranda et al. 2008a, b; Kamboh et al. 2018b). The incidence of AR is a serious concern for human health because the use of antimicrobial agents has been continuously increasing the types, strains, and numbers of antibiotic-resistant bacteria

To cite this paper: Kamboh AA, TA Rind, MA Khan, R Burriro, RA Leghari, KK Malhi (2021). Patterns of antimicrobial and multi drug resistance in E. coli and Salmonella isolates of commercial and non-commercial poultry. Intl J Agric Biol 26:52-59 
(ARB). Since humans eat the poultry products, including meat and eggs, the effectiveness of the antimicrobial medicines which are used in poultry feed, has also reduced in human populations (Asai et al. 2006; Caniça et al. 2019). Since the development of a novel group of antimicrobial agents is really a challenging task (Ikele et al. 2020), hence humans cannot afford their existing antimicrobial drugs to be inefficient to control their diseases. The major reason for the increasing spread of $\mathrm{AR}$ is unawareness of the masses about this problem. Hence research needs to be done to provide conclusive evidence about the prevalence of AR in the sources of human foods, particularly poultry meat.

Microbiota lives in the intestines of poultry birds serve as their essential survivors against invading pathogens. Pathogenic microorganisms, such as Escherichia coli, and Salmonella compete with microbiota and cause diseases to poultry birds. E. coli, a commensal bacterium, is globally considered a major reason for the morbidity and mortality of humans and animals by causing food-borne infections (Miskinyte et al. 2013; Khan et al. 2015). It can survive in several hosts. Its pathogenicity and increase in AR have raised concerns regarding community health and socioeconomic values (Pegues 2005). Moreover, Salmonella is considered a growing threat to human health, as it exhibits highly deadly pathogenic behavior in poultry birds. Moreover, Salmonella contaminates poultry meat and spreads infection in humans through the consumption of infected poultry products (Nair and Johny 2019; Han et al. 2020). Moreover, Salmonella is also a human pathogen that also enjoys poultry as an alternate host. Two major species of Salmonella, viz., Salmonella enterica and $S$. bangori have been reported as sources of foodborne diseases in the US (CDCP 2013). There is a remarkably higher incidence of antibiotic-resistance in the microbiota of chickens grown on diets containing antibiotic drugs as compared to the ones reared without antimicrobial drugs (Zhang et al. 2011). Similarly, lower AR bacteria are generally found in the organically grown poultry birds in contrast to traditionally reared ones (Smith-Spangler et al. 2012). The existing published reports used chicken as the model bird for the prevalence and dissemination of AR in commercial and nonconventional poultry farming systems but investigations in commercial and backyard/wild quails are yet to be published.

The present study, therefore, used two poultry models, i.e., commercial and backyard/wild chickens and quails to elucidate the potential risks of AR. In this study, the incidence of AR was explored in E. coli and Salmonella isolated from intestines and meats of commercial and backyard/wild chickens and quails. The results of this study will be beneficial for the poultry industry to understand the perils of AR in commercial poultry, and provoke new research for the production of antibiotic-free poultry.

\section{Materials and Methods}

\section{Sample collection}

Commercial and backyard/wild chickens and quails were procured from a local live bird market of Hyderabad, Sindh, Pakistan. The birds were assigned to four treatment groups, i.e., C0: backyard chickens, C1: commercial broiler, Q0: backyard/wild quails and Q1: commercial quails. Each treatment group contained twenty birds ( $\mathrm{n}=20$ each). The birds of each treatment group were exsanguinated using a sharp sterilized knife on different days according to the requirements of the Directorate of Advanced Studies, Sindh Agriculture University, Tandojam. The carcasses were eviscerated and portioned using sterilized equipment. The cecal contents of each bird were collected under aseptic conditions and stored in a laboratory freezer at $-24^{\circ} \mathrm{C}$ until required for analysis. The whole carcasses were subject to chilling $\left(4^{\circ} \mathrm{C}\right)$ after slaughter and analyzed for the isolation of pathogenic microbes within $2 \mathrm{~h}$.

\section{Isolation of $\boldsymbol{E}$. coli and Salmonella}

The isolation of two pathogenic microorganisms, including E. coli and Salmonella, from the ceca, was performed by taking $1 \mathrm{~g}$ samples, placing them in $9 \mathrm{~mL}$ of $0.9 \%$ sterile saline solution in a sterilized beaker, and vortexed. The samples were subsequently diluted to prepare ten-fold dilution using the saline solution. About $1 \mathrm{~mL}$ of each 10 fold diluted sample was cultured on the media, as described by Habib et al. (2015).

On the other hand, the whole carcass was hand rinsed under aseptic conditions in $100 \mathrm{~mL}$ of $0.85 \%$ sterile saline solution, according to the method of Kilonzo-Nthenge et al. (2008). About $25 \mathrm{~mL}$ of this solution was taken and mixed with $225 \mathrm{~mL}$ of $0.1 \%$ peptone water. The samples were then incubated at $37^{\circ} \mathrm{C}$ for $24 \mathrm{~h}$ (pre-enrichment culturing).

The sub-culturing of E. coli and Salmonella was done by selecting the suspected bacteria and individually inoculating them onto differential bacteriological agar media (Oxoid, Co., UK) under aseptic conditions. The samples were incubated at $37^{\circ} \mathrm{C}$ for $12 \mathrm{~h}$ and pure cultures were obtained. The conformation of bacterial species was performed according to the method of Monica (1985).

\section{Assessment of antibiotic resistance}

The AR patterns of both the pathogenic isolates were verified according to the protocol described by CLSI Guidelines (2012). Briefly, the AR of the pathogenic bacterial (E. coli and Salmonella) isolates was assessed by the disk dispersion method. The colonies of the pathogenic bacteria were added into nutrient broth. The turbidity ( 0.5 McFarland standard) of the broth was adjusted. These were spread on sensitivity agar plates using sterile swabs. Subsequently, these were dried. In the next step, the selected 
antibiotic discs of the antibiotic drugs were put on the aforementioned sensitivity plates by maintaining an even distance. The amounts of the antibiotic drugs were about 5 $\mu \mathrm{g}$ for ciprofloxacin; $10 \mu \mathrm{g}$ each for ampicillin, gentamicin, and norfloxacin; $25 \mu \mathrm{g}$ for sulfamethoxazole; and $30 \mu \mathrm{g}$ each for chloramphenicol and oxytetracycline. Subsequently, these plates were incubated at $37^{\circ} \mathrm{C}$. The inhibition zones were measured after $24 \mathrm{~h}$ of the incubation from the centre of the disc. The classification of AR breakpoints was done using the protocol published by Lalitha (2004), according to which the bacteria which had an inhabitation zone $\geq 21 \mathrm{~mm}$ were termed as 'Susceptible'; those between 17-20 mm were considered 'Intermediate'; and the ones having $\leq 16 \mathrm{~mm}$ were classified 'Resistants'. Multidrug-resistant (MDR) strains were those whose isolates displayed resistance against 3 or more antibiotics (Kamboh et al. 2018b). The data for these parameters were collected in triplicate.

\section{Statistical analysis}

The general calculations and data analysis were performed using Microsoft Excel (v. 2010). The comparison of the levels of AR between the four treatment groups $(P<0.05)$ was performed by Fisher's exact test using JMP software (5.0.1.a, SAS, USA).

\section{Results}

\section{The incidence of antimicrobial resistance}

The data regarding AR of bacterial isolates obtained from the backyard and commercial chickens and quails against 5 $\mu \mathrm{g}$ ciprofloxacin, $10 \mu \mathrm{g}$ each of ampicillin, gentamicin, neomycin, norfloxacin, $25 \mu \mathrm{g}$ of sulfamethoxazole, and 30 $\mu \mathrm{g}$ each of chloramphenicol and oxytetracycline, and indicated significant differences $(P<0.01)$ with only one exception (Table 1). In this study, the comparison between the treatment groups revealed that the bacterial isolates obtained from both of the backyard chickens and quails exhibited significantly lower levels of AR than those obtained from commercial birds against the eight antimicrobial drugs tested in this study. The bacterial isolates obtained from the $\mathrm{C} 1$ group exhibited the highest AR against each of ampicillin, ciprofloxacin, neomycin, norfloxacin, sulfamethoxazole, and oxytetracycline. In contrast, the bacterial isolates obtained from the Q1 group exhibited the highest AR against each of the chloramphenicol, gentamicin in this study. On the other hand, the comparison between the antibiotic drugs revealed that the highest levels of AR in the bacterial isolates were observed against ampicillin for all the four bird groups. Moreover, the second-worst results were observed against gentamicin in $\mathrm{C} 0$, neomycin in each of $\mathrm{C} 1$ and $\mathrm{Q} 0$, and ciprofloxacin in Q1. Finally, the lowest levels of AR of the bacterial isolates were observed against norfloxacin in each of C0 and Q0, sulfamethoxazole in each of Q0 and Q1, and chloramphenicol in $\mathrm{C} 1$.

\section{The resistance of $E$. coli against antimicrobial drugs}

The percentages of $E$. coli isolated from the intestines of commercial and non-commercial chickens and quails selected in this study were shown in Fig. 1. The criteria described by Lalitha (2004) was used to classify the E. coli resistance against the eight antimicrobial drugs, according to which the pathogen was susceptible to the antibiotic drug if the inhibition zone on the disk was $\geq 21 \mathrm{~mm}$, whereas the pathogen was resistant to the drug if the inhibition zone was: $\leq 16 \mathrm{~mm}$, while the zone between 17-20 mm was considered intermediate. In this study, the comparison between the treatment groups revealed that the $E$. coli isolates of the commercial birds showed significantly higher resistance than the backyard ones against all the tested antimicrobial drugs. Moreover, the E. coli isolates of $\mathrm{C} 1$ exhibited a significantly $(P<0.01)$ higher levels of AR than those of $\mathrm{C} 0$, whereas those of Q1 higher than those of Q0. Furthermore, the E. coli isolates of $\mathrm{C} 1$ exhibited higher levels of AR than those of Q1 against five drugs (ampicillin, neomycin, norfloxacin, sulfamethoxazole, and oxytetracycline), but lower against two drugs (gentamicin and chloramphenicol). On the other hand, the comparison between the antibiotic drugs revealed that the $E$. coli isolates exhibited the highest levels of AR against ampicillin in all the four treatment groups. Finally, the E. coli isolates exhibited the lowest levels of AR against gentamicin in $\mathrm{C} 1$, and against sulfamethoxazole in Q1. However, the E. coli isolates of backyard chickens showed the least resistance against four drugs (ciprofloxacin, neomycin, norfloxacin, and sulfamethoxazole). Finally, the E. coli isolates of backyard quails exhibited no resistance against four drugs (ciprofloxacin, norfloxacin, chloramphenicol, and oxytetracycline) in this study.

\section{The resistance of Salmonella against antimicrobial drugs}

The data regarding the percentages of Salmonella isolates obtained from the intestines of commercial and noncommercial chickens and quails selected in this study were shown in Fig. 2. The isolates obtained from commercial birds exhibited significantly $(P<0.01)$ higher AR than those isolated from non-commercial ones against all the tested antimicrobial drugs. Moreover, the Salmonella isolates of $\mathrm{C} 1$ exhibited significantly higher levels of $\mathrm{AR}$ than those obtained from $\mathrm{C} 0$, whereas those of Q1 higher than those of Q0. Furthermore, the Salmonella isolates of C1 exhibited higher levels of AR than those of Q1 against all the tested antimicrobial drugs in this study. On the other hand, the comparison between the antibiotic drugs revealed that the Salmonella isolates exhibited the highest levels of AR against ampicillin in all the four bird groups. Moreover, the Salmonella isolates exhibited the least of AR against two 
Table 1: Overall percentages of bacterial isolates of commercial and non-commercial poultry resistant to antimicrobial agents

\begin{tabular}{llllllllll}
\hline Antimicrobials & \multirow{2}{*}{$\mu \mathrm{g}$} & & $\mathrm{C} 0 \mathrm{n}=44$ & \multicolumn{2}{c}{$\mathrm{C} 1 \mathrm{n}=64$} & & $\mathrm{Q} 0 \mathrm{n}=44$ & \multicolumn{2}{c}{$\mathrm{Q} 1 \mathrm{n}=56$} \\
\cline { 3 - 10 } & & $\#$ & $\%$ & $\#$ & $\%$ & $\#$ & $\%$ & $\#$ & $\%$ \\
\hline Ciprofloxacin & 5 & 5 & 11.36 & 44 & 68.75 & 1 & 2.27 & 38 & 67.86 \\
Ampicillin & 10 & 14 & 31.82 & 56 & 87.50 & 6 & 13.64 & 44 & 78.57 \\
Gentamicin & 10 & 8 & 18.18 & 35 & 54.69 & 3 & 6.82 & 35 & 62.50 \\
Neomycin & 10 & 6 & 13.64 & 52 & 81.25 & 4 & 9.09 & 34 & 60.71 \\
Norfloxacin & 10 & 3 & 6.82 & 41 & 64.06 & 1 & 2.27 & 34 & 60.71 \\
Sulfamethoxazole & 25 & 5 & 11.36 & 43 & 67.19 & 1 & 2.27 & 32 & 57.14 \\
Chloramphenicol & 30 & 7 & 15.91 & 35 & 54.69 & 2 & 4.55 & 33 & 58.93 \\
Oxytetracycline & 30 & 6 & 13.64 & 49 & 76.56 & 2 & 4.55 & 33 & 58.93 \\
\hline
\end{tabular}

C0: backyard chickens; $\mathrm{Cl}$ : commercial broiler; Q0: backyard/wild quails; Q1: commercial quails ( $P$ Value $<0.001$ for all antimicrobials)

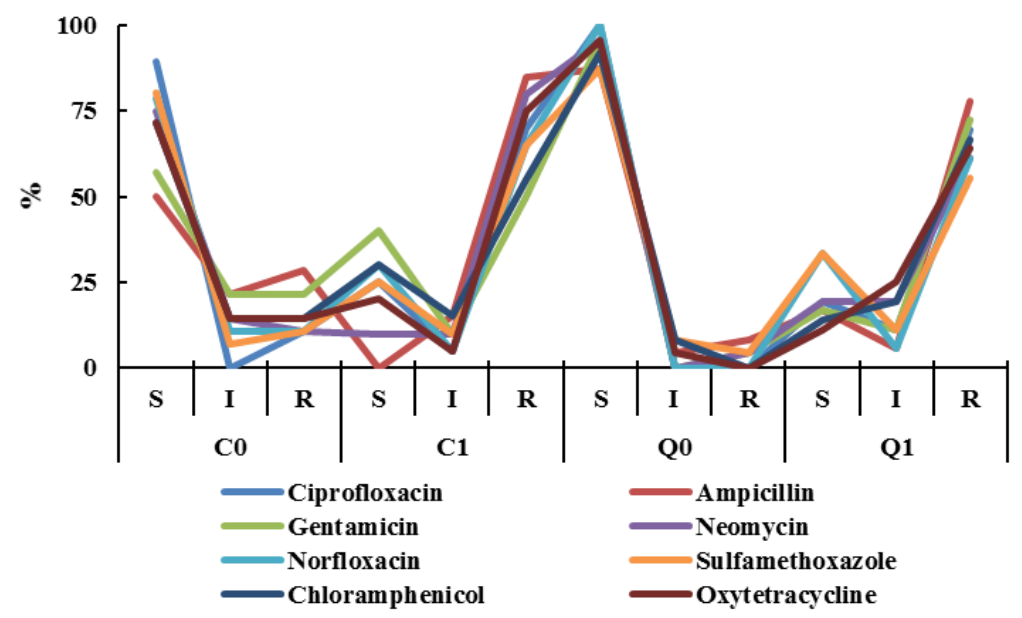

Fig. 1: Percentages of Escherichia coli isolated from commercial and non-commercial poultry susceptible (S), intermediate (I) and resistant $(\mathrm{R})$ to antimicrobial agents by disk diffusion method. C0: backyard chickens ( $\mathrm{n}=28$ ); $\mathrm{C1}$ : commercial broiler ( $\mathrm{n}=40$ ); $\mathrm{Q} 0$ : backyard/wild quails ( $\mathrm{n}=24)$; $\mathrm{Q}$ : commercial quails $(\mathrm{n}=36)$

antimicrobial drugs including gentamicin and chloramphenicol in Q1. Furthermore, the isolates of both the commercial bird types (C1 and Q1) showed the lowest resistance against chloramphenicol. Finally, the Salmonella isolates obtained from backyard chickens and quails exhibited no resistance against norfloxacin and sulfamethoxazole, respectively in this study.

\section{Multidrug resistance patterns}

The data regarding the prevalence of MDR in the two bacterial isolates obtained from commercial and backyard poultry were summarized in Fig. 3. The percentages of MDR E. coli and Salmonella isolates of commercial birds (95 and $77 \%$ for $\mathrm{C} 1$ and Q1) were significantly higher $(P=$ 0.0082 and 0.0076 , respectively) than those obtained from non-commercial ones (67.8 and $54.2 \%$ for $\mathrm{C} 0$ and Q0). Similarly, the levels of MDR Salmonella isolates of commercial birds (92.8 and 85\% for C1 and Q1) were higher $(P=0.0266$ and 0.0060 , respectively) than their counterparts obtained from the backyard birds (81.2 and $60 \%$ for $\mathrm{C} 0$ and Q0). The best results were shown by Q0, for which 45.8 and $40 \%$ E. coli and Salmonella isolates showed the resistance against $0-1$ antimicrobial drugs, whereas the worst results were shown by $\mathrm{C} 1$, for which 42.8 and $28.6 \%$ E. coli and Salmonella isolates showed the resistance against $>6$ antimicrobial drugs.

\section{Discussion}

Food-borne pathogens have become a leading threat to public health throughout the world. These include E. coli, Salmonella, Shigella, and Campylobacter, while raw and partially-cooked poultry meat products harbor them the most. Particularly, Salmonella and E. coli proliferate to human populations during the handling of untreated poultry carcasses, as well as through consumption of partially or improperly cooked poultry products (Chen et al. 2020). Another rising threat to human beings is the incidence of AR microorganisms, for which leading cause has been thought to be the non-judicious use of antibiotic drugs in the poultry feed. A recently published report suggested that in the European countries the E. coli isolates obtained from poultry and cattle exhibited greater resistance to antimicrobial drugs, and there were very strong associations between the dosages of antibacterial drugs and their patterns of antibiotic resistance (Chantziaras et al. 2013; Ariffin et al. 2019). Moreover, FDA has highlighted that the resistance against antimicrobial drugs in the Enterobacteriaceae family can be transferred from poultry 


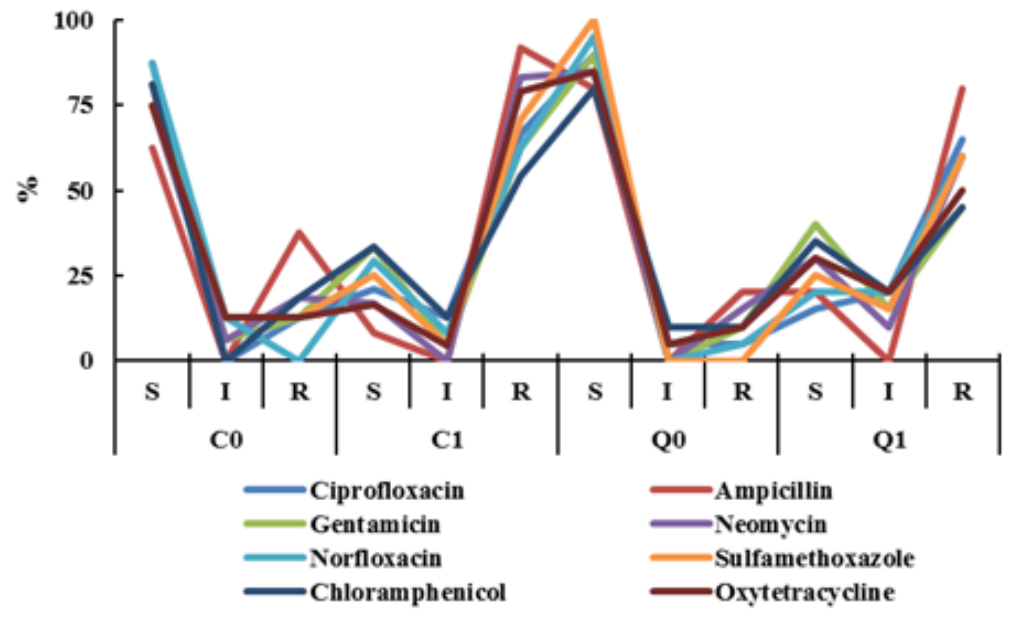

Fig. 2: Percentages of Salmonella isolated from commercial and non-commercial poultry susceptible (S), intermediate (I) and resistant (R) to antimicrobial agents by disk diffusion method. C0: backyard chickens ( $\mathrm{n}=16) ; \mathrm{Cl}$ : commercial broiler ( $\mathrm{n}=24)$; Q0: backyard/wild quails ( $\mathrm{n}=20)$; Q1: commercial quails $(\mathrm{n}=20)$

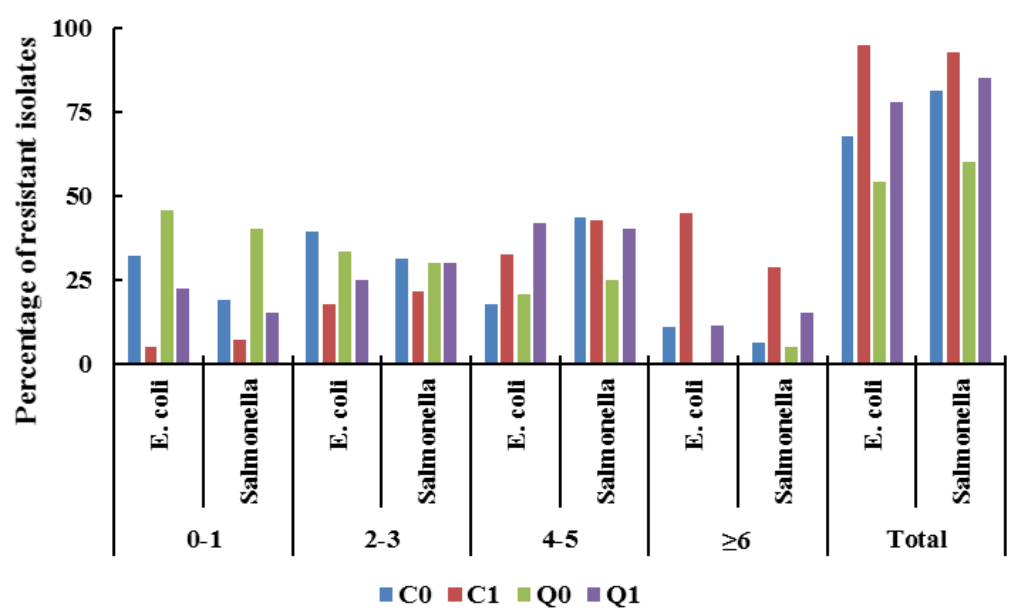

Fig. 3: Percentage of Escherichia coli and Salmonella isolates of commercial and non-commercial poultry resistant to multiple antimicrobials. C0: backyard chickens; C1: commercial broiler; Q0: backyard/wild quails; Q1: commercial quails

reared on those antimicrobial drugs to human populations (Rafiei and Nasirian 2003), particularly through the consumption of eggs, milk, and meat obtained from such animals and poultry birds (Reig and Toldra 2008).

The primary purpose of using antibiotic drugs in poultry feed and water is to prevent diseases in the commercial flocks. However, owing to the enhanced feed efficiency and weight gain with these drugs, their nonjudicious use up to unacceptable levels has increased to achieve maximum monetary benefits from the commercial poultry flocks. Recently published reports have suggested that the non-medical use of these drugs results in rendering these drugs ineffective against the control of few strains of gut microbes, which upon poultry harvest can be transferred to the human food chain (Koga et al. 2015). Our previously results (Kamboh et al. 2018b) and findings of some other authors (Miranda and Zemelman 2002; Nisar et al. 2017; Davis et al. 2018; Gad et al. 2018) suggest that the incidence of AR microbes was higher in commercially reared chickens as compared with those in domestically reared ones. In this study, the E. coli and Salmonella isolates obtained from the commercial broiler chickens and quails exhibited alarmingly higher resistance patterns against the eight antibiotic drugs tested in this experiment, as compared with the isolates obtained from backyard chickens and wild quails. In previously published studies, poultry reared without antibiotics reported less antibiotic-resistant $E$. coli as compared with the conventionally reared ones (Zhang et al. 2011). Similarly, the chicks reared on an organic diet have also been found to have lower numbers of antibioticresistant bacteria than those reared on a commercial diet (Smith-Spangler et al. 2012).

Unfortunately, during the last two decades, the nonjudicial use of antibiotics for seeking higher profitability in the poultry sector has tremendously increased in most of the countries having poor economic conditions. Particularly 
in Pakistan, the increase in the use of antibiotics has been about $65 \%$ in the last 16 years in the poultry sector (Klein et al. 2018). The major reason associated with nonjudicial infeed consumption of antibiotics for poultry production includes poor rearing conditions, high microbial contamination in feed and drinking water, imbalanced poultry nutrition, increasing occurrences of urbanizationrelated nonbacterial infections, which have caused to increased prevalence of AR of pathogenic bacteria (Weaver 2013; Collineau et al. 2017; Nandi et al. 2017; Klein et al. 2018). Particularly, in this report, all the bacterial isolates showed high resistance (about 54\%) against chloramphenicol. It is an antibiotic drug that has been banned for use in the poultry diet in several countries such as Europe and the US; however, it is still excessively being used in several developing countries.

Moreover, the findings of this study revealed that the commercial broiler chickens and quails exhibited a higher incidence of MDR strains of both E. coli and Salmonella than those of non-commercial chickens and quails. These findings agreed to the results of Miranda et al. (2008a), who found a higher incidence of MDR enteric microbes in the meat obtained from commercially reared chickens $(63.3 \%)$ as compared with those obtained from turkeys $(56.7 \%)$ and organic chickens (41.7\%) after the application of aminoglycosides, penicillins, and quinolones in the diets of commercial chicks. Extensive and non-judicial use of antibacterial agents in the poultry feeds makes Salmonella and $E$. coli become resistant against those drugs (Fielding et al. 2012). The phenomenon of MDR against the antimicrobial drugs investigated in this study was in agreement with the earlier published report (Brown et al. 2019).

Several studies have investigated the role of antimicrobial drugs in the poultry production systems in the induction of AR in poultry birds (Chantziaras et al. 2013; Kamboh et al. 2018b). Moreover, several reports have published the resistance patterns of pathogenic microbial isolates obtained from poultry being reared in the organic and conventional production systems (Miranda et al. 2008b; Miranda et al. 2009). It has been well documented that the zoonotic microorganisms isolated from conventional poultry production systems exhibit a remarkably higher prevalence of $\mathrm{AR}$ in contrast to the ones isolated from organic production systems (Young et al. 2009). Particularly, the isolates of $E$. coli obtained from conventional and organic production systems exhibited similar AR patterns in a European study (Österberg et al. 2016). Similarly, some other reports also found that the isolates of E. coli and Staphylococcus aureus from beef (Miranda et al. 2009) and intestines (Sato et al. 2004, 2005) obtained from conventional rearing system exhibited higher antimicrobial resistance in contrast to the ones obtained from an organic production system. Furthermore, the phenomenon of multidrug resistance was also higher in the bacterial isolates of the liver obtained from commercial broilers as compared to those of backyard chickens (Kamboh et al. 2018b).

\section{Conclusion}

The AR of both the bacterial isolates (E. coli and Salmonella) obtained from commercial broiler chickens and quails were higher in contrast to those of their noncommercial counterparts against the eight antimicrobial drugs tested in this study. Moreover, the total bacterial ( $E$. coli and Salmonella) isolates obtained from all the four bird groups were the most resistant against ampicillin, followed by neomycin in this study. Finally, the incidence of MDR in both bacteria was also higher in the commercial chickens and quails isolates as compared with their non-commercial backyard counterparts.

\section{Acknowledgements}

Authors are highly thankful to Central Veterinary Diagnostic Laboratory (CVDL) Tandojam for providing facilities to carry out this work.

\section{Authors Contributions}

Asghar Ali Kamboh conceptualized the study, Asghar Ali Kamboh and Tarique Ali Rind performed the research. Muhammad Amar Khan wrote the manuscript, and Kanwar Kumar Malhi contributed to analysis and interpretation of the study data. Rehana Burriro and Riaz Ahmed Leghari helped in analysis and proof reading.

\section{Conflict of interest}

The authors declare that they have no conflict of interest.

\section{Data availability}

All data reported in this article are available and will be produced on demand.

\section{Ethics Approval}

All study protocols were approved by the Directorate of Advanced Studies, Sindh Agriculture University, Tandojam.

\section{References}

Ariffin SMZ, N Hasmadi, NM Syawari, MZ Sukiman, TAM Faiq, MH Chai, MF Ghazali (2019). Prevalence and antibiotic susceptibility pattern of Staphylococcus aureus, Streptococcus agalactiae and Escherichia coli in dairy goats with clinical and subclinical mastitis. J Anim Health Prod 7:32-37

Asai T, H Esaki, A Kojima, K Ishihara, Y Tamura, T Takahashi (2006). Antimicrobial resistance in Salmonella isolates from apparently healthy food-producing animal from 2000 to 2003: The first stage of Japanese Veterinary Antimicrobial Resistance Monitoring (JVARM). J Vet Med Sci 68:881-884

Brown EEF, A Cooper, C Carrillo, B Blais (2019). Selection of multidrugresistant bacteria in medicated animal feeds. Front Microbiol 10; Article 456 
Caniça M, V Manageiro, H Abriouel, J Moran-Gilad, CMAPFranz (2019). Antibiotic resistance in foodborne bacteria. Trends Food Sci Technol $84: 41-44$

CDCP (2013). Center for disease control and prevention. Listeria (Listeriosis). Retrieved from http://www.cdc.gov/listeria/statistics.html\#one

Chantziaras I, F Boyen, B Callens, J Dewulf (2013). Correlation between veterinary antimicrobial use and antimicrobial resistance in foodproducing animals: A report on seven countries. J Antimicrob Chemother 69:827-834

Chen SH, N Fegan, C Kocharunchitt, JP Bowman, LL Duffy (2020). Changes of the bacterial community diversity on chicken carcasses through an Australian poultry processing line. Food Microbiol 86; Article 103350

CLSI Guidelines (2012). Clinical and Laboratory Standards Institute Standards Development Policies and Process. Annapolis Junction, Maryland, USA

Collineau L, C Belloc, KDC Stärk, A Hémonic, M Postma, J Dewulf, C Chauvin (2017). Guidance on the selection of appropriate indicators for quantification of antimicrobial usage in humans and animals. Zoonos Publ Health 64:165-184

Davis GS, K Waits, L Nordstrom, H Grande, B Weaver, K Papp, J Horwinski, B Koch, BA Hungate, CM Liu, LB Price (2018). Antibiotic-resistant Escherichia coli from retail poultry meat with different antibiotic use claims. BMC Microbiol 18; Article 174

Fielding BC, A Mnabisa, PA Gouws, T Morris (2012). Antimicrobialresistant Klebsiella species isolated from free-range chicken samples in an informal settlement. Arch Med Sci 8:39-42

Gad AH, UH Abo-Shama, KK Harclerode, MK Fakhr (2018). Prevalence, serotyping, molecular typing, and antimicrobial resistance of Salmonella isolated from conventional and organic retail ground poultry. Front Microbiol 9; Article 2653

Habib F, KK Malhi, AA Kamboh, R Rind, R Burriro (2015). Antimicrobial susceptibility profile of Staphylococcus aureus isolates recovered from various animal species. J Anim Health Prod 3:99-103

Han X, J Peng, X Guan, J Li, X Huang, S Liu, Y Wen, Q Zhao, X Huang, Q Yan, Y Huang, S Cao, R Wu, X M, L Zou (2020). Genetic and antimicrobial resistance profiles of Salmonella spp. isolated from ducks along the slaughter line in southwestern China. Food Cont 107; Article 106805

Ikele OM, IM Ezeonu, CN Umeh (2020). Prebiotic roles of Ocimum gratissimum extract in the control of colibacillosis in broilers. J Anim Health Prod 8:206-211

Kamboh AA, MA Khan, U Kaka, EA Awad, AM Memon, M Saeed, NA Korejo, M Bakhetgul, C Kumar (2018a). Effect of dietary supplementation of phytochemicals on immunity and haematology of growing broiler chickens. Ital J Anim Sci 17:1038-1043

Kamboh AA, M Shoaib, SH Abro, MA Khan, KK Malhi, S Yu (2018b). Antimicrobial resistance in Enterobacteriaceae isolated from liver of commercial broilers and backyard chickens. J Appl Poult Res 27:627-634

Khan A, R Rind, M Shoaib, AA Kamboh, GA Mughal, SA Lakho, KK Malhi, AR Nizamani, A Yousaf (2015). Isolation, identification and antibiogram of Escherichia coli from table eggs. J Anim Sci Heath Prod 4:1-5

Klein EY, TPV Boeckel, EM Martinez, S Pant, S Gandra, SA Levin, H Goossens, R Laxminarayan (2018). Global increase and geographic convergence in antibiotic consumption between 2000 and 2015. Proc Natl Acad Sci USA 115:3463-3470

Kilonzo-Nthenge A, SN Nahashon, F Chen, N Adefope (2008). Prevalence and antimicrobial resistance of pathogenic bacteria in chicken and Guinea fowl. Poult Sci 87:1841-1848

Koga VL, S Scandorieiro, EC Vespero, A Oba, BGD Brito, KCD Brito, G Nakazato, RK Kobayashi (2015). Comparison of antibiotic resistance and virulence factors among Escherichia coli isolated from conventional and free-range poultry. BioMed Res Intl 2015; Article 618752

Lalitha MK (2004). Manual on antimicrobial susceptibility testing. In: Performance standardsforantimicrobial testing: Twelfth Informational Supplement, pp:454-456. NCCLS, Wayne, Pennsylvania, USA
Miranda CD, R Zemelman (2002). Antimicrobial multiresistance in bacteria isolated from freshwater Chilean salmon farms. Sci Tot Environ 293:207-218

Miranda J, A Mondragón, B Vázquez, C Fente, A Cepeda, C Franco (2009). Influence of farming methods on microbiological contamination and prevalence of resistance to antimicrobial drugs in isolates from beef Meat Sci 82:284-288

Miranda J, M Guarddon, B Vázquez, C Fente, J Barros-Velázquez, A Cepeda, C Franco (2008a). Antimicrobial resistance in Enterobacteriaceae strains isolated from organic chicken, conventional chicken and conventional turkey meat: A comparative survey. Food Cont 19:412-416

Miranda J, B Vazquez, C Fente, P Calo-Mata, A Cepeda, C Franco (2008b). Comparison of antimicrobial resistance in Escherichia coli, Staphylococcus aureus, and Listeria monocytogenes strains isolated from organic and conventional. Poult Meat J Food Prot 71:2537-2542

Miskinyte M, A Sousa, RS Ramiro, JAD Sousa, J Kotlinowski, I Caramalho, S Magalhães, MP Soares, I Gordo (2013). The genetic basis of Escherichia coli pathoadaptation to macrophages. PLoS Pathog 9; Article e1003802

Monica C (1985). Medical laboratory manual for tropical countries. Trop Health Technol 42:250-290

Nair DV, AK Johny (2019). Salmonella in poultry meat production. In: Food Safety in Poultry Meat Production, pp:1-24. Springer, Nature, Switzerland

Nandi A, I Megiddo, A Ashok, A Verma, R Laxminarayan (2017). Reduced burden of childhood diarrheal diseases through increased access to water and sanitation in India: A modeling analysis. Soc Sci Med 180:181-192

Nisar M, II Kassem, G Rajashekara, SM Goyal, D Lauer, S Voss, KV Nagaraja (2017). Genotypic relatedness and antimicrobial resistance of Salmonella Heidelberg isolated from chickens and turkeys in the midwestern United States. J Vet Diagnos Invest 29:370-375

Obajuluwa OV, KA Sanwo, LT Egbeyale, AO Fafiolu (2021). Carcass characteristics and meat quality of broiler chicken fed diets supplemented with Pausynistalia yohimbe and larvicide (Cyromazine). J Anim Health Prod 9:40-46

Österberg J, A Wingstrand, AN Jensen, A Kerouanton, V Cibin, L Barco, M Denis, S Aabo, B Bengtsson (2016). Antibiotic resistance in Escherichia coli from pigs in organic and conventional farming in four European countries. PLoS One 11; Article e 0157049

Pegues DA, ME Ohl, SI Miller (2005). Salmonella species including Salmonella typhi. In: Principles and practice of infectious diseases $6^{\text {th }}$ edn, pp:2636-2654. Bennett J, R Dolin, MJ Blaser (Eds). Elsevier Churchill Livingstone, Philadelphia, USA

Rafiei TR, A Nasirian (2003). Isolation, identification and antimicrobial resistance patterns of E. coli isolated from chicken flocks. Iran J Pharmacol Therapeut 2:39-30

Reig M, F Toldrá (2008). Veterinary drug residues in meat: concerns and rapid methods for detection. Meat Sci 78:60-67

Sato K, PC Bartlett, MA Saeed (2005). Antimicrobial susceptibility of Escherichia coli isolates from dairy farms using organic versus conventional production methods. J Amer Vet 226:589-594

Sato K, T Bennedsgaard, P Bartlett, R Erskine, J Kaneene (2004). Comparison of antimicrobial susceptibility of Staphylococcus aureus isolated from bulk tank milk in organic and conventional dairy herds in the midwestern United States and Denmark. J Food Prot 67:1104-1110

Schwarz S, C Kehrenberg, T Walsh (2001). Use of antimicrobial agents in veterinary medicine and food animal production. Intl J Antimicrob Agents 17:431-437

Smith-Spangler C, ML Brandeau, GE Hunter, JC Bavinger, M Pearson, PJ Eschbach, V Sundaram, H Liu, P Schirmer, C Stave, I Olkin (2012). Are organic foods safer or healthier than conventional alternatives? A systematic review. Ann Intern Med 157:348-366

Weaver SC (2013). Urbanization and geographic expansion of zoonotic arboviral diseases: mechanisms and potential strategies for prevention. Trends Microbiol 21:360-363 
Young I, A Rajić, BJ Wilhelm, L Waddell, S Parker, SA McEwen (2009). Comparison of the prevalence of bacterial enteropathogens, potentially zoonotic bacteria and bacterial resistance to antimicrobials in organic and conventional poultry, swine and beef production: a systematic review and meta-analysis. Epidemiol Infect 137:1217-1232
Zhang J, A Massow, M Stanley, M Papariella, X Chen, B Kraft, P Ebner (2011). Contamination rates and antimicrobial resistance in Enterococcus spp., Escherichia coli, and Salmonella isolated from "No antibiotics added"-labelled chicken products. Foodborne Pathog Dis 8:1147-1152 